\title{
A Brief Overview of the Discussion on Universal Grammar with a Focus on Chomsky's Theory
}

\section{Renxiu Liu}

Zhongyuan University of Technology, School of Foreign Languages

\begin{abstract}
Over the past decades, linguists have argued over whether language abilities can be described as universal, something common to all speakers, or, whether they are learned secondarily. This paper introduces the main arguments for each side and arrives at a conclusion in support of universal language.
\end{abstract}

Keywords: universal grammar, Chomsky's theory

\section{Introduction}

In her study where infants were exposed to sound sequences, Saffran observed that infants were distracted for a longer time period by unfamiliar sound sequences than by sounds they were preconditioned to. She concluded that this is because humans have an innate ability to distinguish sounds, filter the sounds and pauses (Saffran). The idea that certain sounds seem to connect is the result of universal grammar that underlies all language. Regarding the language, linguist Noam Chomsky claims that since language is innate, there is a competence native to all speakers and hence universal grammar is shaped because the competence to distinguish sounds and ordering is universally shared by all speakers. However, Fodor and Garrett argues against the positivist approach and asserts that positivists abstract away too much of the variations in language and does not account for the underlying reasoning for all the variations. This paper discusses these views in details.

\section{Chomsky's Theory}

To illustrate his emphasis on innate competence of humans, Chomsky first introduces competence and performance. According to Chomsky, linguistic competence is the ability of the idealized speaker-hearer to associate sounds and meanings strictly in accordance with the rules of his language" (Chomsky 398). Therefore it is evident that Chomsky"s definition of competence refers to a broad and deep understanding of language, not specifically how language is spoken by each individual. Such specifics, such as variations in pronunciation, is referred to as performance. However, "Linguistic performance is, furthermore, governed by principles of cognitive 
structure...that are not, properly speaking, aspects of language" (Chomsky 398). Therefore, to Chomsky, the varying performance can be abstracted away when studying language since it represents personal difference and does not truly represent variations in language.

When the variations among individual performances are abstracted, what is left of language is the inherent linguistic competence. This accounts for the similarities in languages that humans use. To evidence the claim, Chomsky lists sentences that are in the active and passive voice. Although sentences have different surface structures, the listener can still distinguish the sentences and understand that they mean the same thing. According to Chomsky, this is because "The grammar that each speaker has internalized does distinguish these deep 2 structures...But this fact about his internalized grammatical competence may escape even the careful attention of the native speaker" (Chomsky 433). In other words, since internalization is so universal, speakers can overlook the varying performances and accept universal grammar as the reason for the shared understanding.

\section{Additional Theories}

While Fodor and Garrett acknowledge that some slight variations in performance are individualistic and can be abstracted away from competence, they also believe that to simply take away the differences in over simplistic. This is because while certain variations are merely variations, other variations are results of deeper differences among human speech. And the positivists would overlook such distinctions because they tend to study the evidence of language rather than the underlying rules. More specifically, the "evidence" is that there are variations among individuals of the same language. Positivists would assume that all is presented is evidence, evidence of competence rather than presupposition for performance. However, in doing so, they undermine the potential underlying phenomenon that caused such variations in performance. That abstracted underlying phenomenon may be the root of scientific study in the field. Therefore, the positivists, in their approach of language, overlook "preposition p" and focus excessively on "the evidence for p" (Fodor and Garrett 135). For example, if the linguist "held that the object of his study was literally the behavior of speakers, his data was impoverished ..." (Fodor and Garrett 137). This is because if linguists study the behavior alone, then they are studying the evidence alone, without venturing into the actual preposition that caused such evidence to occur. Therefore, there may be underlying differences in language, as presented by variation in performance, but since positivists study the evidence alone and disregard the differences in performance, they have chosen to only accept the competence aspect of their studies. This is why the linguist"s data "is impoverished;" the linguist has chosen to ignore the prepositions for variations in performance and only accept competence. 


\section{Discussion}

Although Fodor and Garrett"s counterargument is well-supported, I nevertheless agree with Chomsky regarding innate linguistic competence. For example:

John ate an apple. (A)

An apple was eaten by John. (B)

Although A and B are syntactically different, they nevertheless represent the same idea. The fact that we can interpret the different sentences to mean the same thing implies that we are innately able to deemphasize the details and focus on the true universal grammar. We can inherently interpret both sentences to mean the same thing because B"s grammar is not diametrically different from that of A. In fact, B has "John" and "an apple" switched and the tense "ate" to "was eaten." But ultimately, since B can be transformed into A, we can interpret B in the form of A: that the person John ate an object, he ate an apple.

\section{Conclusion}

Based on the above overview, the author believes in universal grammar because similar grammar applies to languages such as Chinese as well. Although both A and B can be translated into Chinese, the A structure is prevalent (subject, verb, noun). Despite the differences in culture, history, and phonology, both languages share the subject-verb-noun grammatical formation. Although citing the example of Chinese alone is not enough to prove Chomsky"s theory correct, the example nevertheless does not disprove the theory and helps to show merits of Chomsky"s theory.

\section{References}

[1] Chomsky, Noam. 1967. The formal nature of language. In Biological foundations of language, ed. Eric H. Lenneberg.

[2] John Wiley and Sons, Inc. Fodor, Jerry A., and M. Garrett. 1966. Some reflections on competence and performance. In Psycholinguistics papers, ed. J. Lyons and R. Wales. Edinburgh University Press. Jenny R. Saffran; Richard N. Aslin, and Elissa L. Newport. Statistical Learning by 8-Month Old Infants. In Science, New Series, Vol.274, No.5294. (1996). 